\title{
Naciones Unidas, el Consejo de Seguridad y el Orden Mundial en Formación*
}

\author{
Ronaldo Sardenberg
}

Si bien existe una dicotomía entre los principios de la Carta de 1945 y la realidad, Naciones Unidas es el único mecanismo universal para solucionar controversias y garantizar la paz internacional. Sin embargo, el nuevo entorno internacional exige realizar un examen crítico de su rol en el pasado que le ayude a enfrentar los grandes desarios y posibilite el tránsito de la humanidad hacia un nuevo orden mundial. En virtud de lo anterior, el autor da a conocer su visión y la de su país -Brasil-acerca del futuro de este organismo, especialmente en lo que se refiere a la reestructuración del Consejo de Seguridad y la revitalización de la Asamblea General.

Brasil es uno de los miembros fundadores de Naciones Unidas. Participó, con el sacrificio de sus hijos, en la lucha contra la tiranía nazi-fascista. Estuvo representado en San Francisco, cuando llegó a ser considerado como uno de los posibles miembros permanentes del Consejo de Seguridad. Ha participado siempre activamente en las grandes deliberaciones multilaterales. Por todo eso nos sentimos cómodos para revisar el desempeño histórico de Naciones Unidas, evaluar su rol actual en el mantenimiento de la paz de la seguridad y examinarlo desde el ángulo del futuro del orden internacional.

\footnotetext{
"El texto es una adaptación de la Conferencia que el autor hizo en la Universidad Federal de Pernambuco, en la ciudad de Recife, Brasil, con ocasión del 50 aniversario de Naciones Unidas.
} 
Para todas las naciones nada es más importante que la paz. En Brasil, porque hace más de un siglo hemos vivido en paz con nuestros vecinos, tal vez nos hemos acostumbrado a considerarla un dato inmutable. En verdad, desde el establecimiento de las Naciones Unidas, el mundo ha vivido bajo la constante amenaza del terror nuclear y hemos convivido con la realidad de conflictos en todas las regiones. Hoy en dia la amenaza nuclear está mitigada, pero no ha desaparecido. Los arsenales nucleares son mayores y más numerosos que en 1968 cuando se firmó el Tratado de No-Proliferación de Armas Nucleares.

La cuestión de la paz internacional tiene nuevos contornos. Es más que la ausencia de la guerra nuclear y que la reducción de la amenaza que ella representa. Los conflictos convencionales han proliferado y la paz, para ser verdadera, tendrá que incorporar otras dimensiones.

Si uno la mira desde las atribuciones de Naciones Unidas, la cuestión de la paz no puede ser tomada aisladamente. La verdadera paz, así como la seguridad verdadera, se inscribe en un plano abarcador donde están las dimensiones actuales y futuras del bienestar de los pueblos (con la extrema desigualdad que lo caracteriza), además de las consideraciones de justicia y de democracia que son igualmente esenciales en los planos interno y externo.

Naciones Unidas es permeable a la realidad del cuadro internacional. Ella refleja las imposiciones del poder, o bien las modera y modula. El multilateralismo en cuanto a ser un ejercicio parlamentario internacional es una técnica de trabajo tan valiosa como las relaciones bilaterales. Agrega a esta última un tipo de negociación en que los intereses, los más variados, están simultáneamente representados por los Estados participantes.

Mal o bien, Naciones Unidas constituye la institución política intergubernamental más acabada que la comunidad internacional ha logrado construir en nuestros días. Pero con el final de la Guerra Fría, luego de décadas de confrontación, Naciones Unidas enfrenta nuevos desafios como la idea de que un nuevo orden mundial está en construcción o que la sociedad contemporánea está en tránsito hacia la postmodernidad - un mundo distinto del inaugurado por el iluminismo que prevaleció en este último siglo y medio.

Es necesario, por lo tanto, ubicar a la Organización en el cuadro de la macroestructura intemacional que se dibuja últimamente se discute la viabilidad de Naciones Unidas como la institución que conocemos, dirigida idealmente para la paz, la seguridad colectiva y la armonización 
de las acciones entre los Estados, así como para el progreso de los pueblos en su sentido más amplio.

Ya en 1963, el Canciller brasileño Joao Augusto de Araújo Cástro defendía en la Asamblea General de Naciones Unidas el ideario de las 3 D: Desarme, Desarrollo y Descolonización. Tres décadas más tarde, como Representante Permanente de Brasil junto a Naciones Unidas he tenido la oportunidad de reafirmar el compromiso, firme y continuado, de Brasil con las $3 \mathrm{D}$ que hoy se pueden leer como el Desarrollo, el Desarme y la Democratización de las relaciones intemacionales.

Las dos formulaciones pertenecen al mismo orden de preocupaciones. Tienen por objetivo dar una respuesta doctrinaria y política a momentos políticos y doctrinarios distintos. No nos olvidemos que Naciones Unidas es un organismo vivo que reacciona a las mutaciones del escenario intermacional y que sí es, a veces, lento en su reacción; otras, es capaz de anticipar esos cambios e incluso promoverlos.

El comportamiento de la Asamblea General y del Consejo de Seguridad, en distintos momentos y respecto a temas diversos, ilustra bien las diferencias de foco en la actuación de Naciones Unidas (e incidentalmente de sus expresiones institucionales) en décadas anteriores y hoy dia.

En la lucha por la descolonización y contra el régimen racista del apartheid, Naciones Unidas fue la vanguardia que abrió camino, en el plano intemacional, para las victorias que lograron al final los pueblos que luchaban por su emancipación nacional y por la igualdad racial. Brasil estuvo presente en la creación de los Estados que surgieron del régimen colonial y en la afirmación de la nueva y democrática Africa del Sur. Brasil participó en los grandes embates diplomáticos en ese proceso $y$, a pesar de las dificultades internas que vivió, supo, en los momentos críticos, tomar posiciones que nos enorgullecen. Creo que eso ilustra la madurez del país y la autenticidad de su política externa, además del desarrollo de una visión política y de un compromiso universal.

En todas esas cuestiones la Asamblea General fue más dinámica y actuante que el Consejo de Seguridad, que estuvo básicamente paralizado, por décadas, por las imposiciones de la confrontación Este-Oeste y por la tenaz resistencia de las antiguas metrópolis en tratar los temas coloniales. Brasil mismo, en los años sesenta, tuvo su ámbito de acción en el Consejo de Seguridad limitado por esas imposiciones, De vuelta al Consejo, en 1988-89 y en 1993-94, tuvo por delante un escenario mucho más complejo y fluido, en el cual los parámetros del poder pasaban por un proceso de desconstrucción mientras se buscaban las nuevas fundaciones para el orden internacional. 
Pero ese examen retrospectivo del rol de Naciones Unidas pone en relieve una serie de cuestiones todavía actuales: la imbricación de $\mathrm{Na}$ ciones Unidas respecto del orden contemporáneo; el equilibrio político entre sus organismos principales; la composición y la capacidad de representación de esos organismos; la evolución de las modalidades de actuación de la Organización; y, en suma, la importancia política de Naciones Unidas a largo plazo.

A pesar del inevitable hiato entre intención y realidad, la Carta de Naciones Unidas, sucesora del Pacto de la Liga de las Naciones, creó, como ya señalaba el Embajador Araújo Castro, la única instancia política de carácter universal de que dispone la humanidad para dirimir controversias y garantizar la paz internacional. Aunque exista una tensión institucional entre la Asamblea y el Consejo, puesto que éste tiende todavía a reflejar la relación de fuerzas existente en 1945, es fundamental retener que la Carta de las Naciones Unidas recoge premisas esenciales del orden internacional, que se perfeccionaban por más de tres siglos, como la unidad del sistema intemacional, la pluralidad de los Estados soberanos y la necesidad de la acción colectiva para la cooperación en la paz y en la guerra. Al mismo tiempo, la Carta incorpora, expresamente, los principios de la igualdad de derechos y de la autodeterminación de los pueblos, la igualdad soberana de los Estados y la no-injerencia en.sus asuntos internos. Esas premisas y esos principios son factores extremadamente importantes para la estabilidad internacional, por más que se los cuestione, como parece estar de moda hoy día.

Desde 1945, la Carta logró acomodar el proceso de universalización radical de Naciones Unidas, una conquista política de extraordinario valor y que, por si sola, define también el mundo en que vivimos. En su origen, la Organización contaba con una composición razonablemente homogénea de 51 miembros, la mayoría de los cuales perteneciente a las regiones más desarrolladas del planeta y tenía como miembros un gran número de países fundamentalmente identificados con los valores centrales del sistema. Hoy día, con sus 185 Estados miembros que le dan una configuración casi universal, las diferencias culturales son más sensibles y el sustrato común más tenue, de lo que resulta más dificil la amalgación parlamentaria.

Por otra parte, el Consejo de Seguridad estaba, en su origen, formado en la proporción de cinco a uno respecto al universo de los miembros de la Organización y tenía, así, once miembros, cinco de los cuales permanentes. Al inicio de la década de los sesenta, cuando la Asamblea llegaba a casi cien miembros, su composición fue ampliada a quince, el doble de 
su composición original, con la incorporación acrecimo de los países recién descolonizados.

El Consejo mantiene hasta hoy esa misma formación de quince miembros, pero el proceso de universalización de Naciones. Unidas sigue. En los últimos cinco años, treinta nuevos miembros ingresaron en la Organización, lo que significó a corto plazo un aumento de $19 \%$. La mitad de los nuevos miembros son países europeos y cuatro de ellos tienen dimensiones mínimas: Andorra, San Marino, Mónaco y Liechtenstein. Esos son hechos políticos nuevos que, a lo mejor, no han sido aún asimilados por las estructuras de la Organización.

Desde su establecimiento, Naciones Unidas se ha beneficiado de la convicción de que, a pesar de las concesiones hechas a los dictados del poder (o tal vez incluso por causa de esas concesiones), se había logrado una solución política razonablemente equilibrada para el problema del orden internacional. Las realidades estratégicas y las aspiraciones políticas coexisten, de una manera hasta cierto punto dinámica, en Naciones Unidas. O sea: la Organización provee un foro y garantiza normas que abren espacio político y jurídico para que las aspiraciones se expresen y tenga curso su discusión, sin perjuicio del peso de las realidades dominantes.

Por esa razón, Naciones Unidas ha sido siempre vista como algo más significativo e importante que un simple reflejo de las relaciones de poder y ha podido abrigar -como ya se observó arriba- las luchas por la descolonización y contra el apartheid, aun cuando esas relaciones de poder eran claramente desfavorables a las antiguas colonias y a la población negra en Africa del Sur. Por la misma razón, en la década de los cincuenta, para los países pobres fue posible inaugurar el debate mundial sobre la cuestión del subdesarrollo, que llevó al extraordinario esfuerzo multilateral de la década siguiente en los temas del comercio y del desarrollo. La Asamblea General de Naciones Unidas fue el foro por excelencia para todas esas cuestiones.

De este modo resultan reforzadas tres percepciones centrales. En primer lugar, que la política practicada en Naciones Unidas es parte de algo mayor -la política internacional-y que logra asumir características progresistas siempre que responda a las aspiraciones de la mayoría de la humanidad.

La segunda percepción es que, bajo ciertas circunstancias y en crisis especificas, la Organización puede, y al mismo tiempo debe desde el punto de vista de su constitución, tener una actuación decisiva en la obtención de soluciones políticas que sean colectivamente aceptables. 
En efecto, Naciones Unidas, como instancia del sistema internacional, compite por la atención mundial con los principales y más poderosos de sus Estados miembros -a quienes los caminos unilaterales suelen presentarse a veces más atractivos que el sendero multilateral-, además de competir con otras entidades y organismos. Entre esos últimos, el GATT (hoy díala Organización Mundial de Comercio), las entidades que integran un tanto en teoría la llamada "familia de Naciones Unidas" (como los organismos financieros de Bretton Woods; el Banco Mundial y el Fondo Monetario Internacional), u organizaciones de composición y ámbito geográfico más restrictivos, como la Organización del Tratado del Atlántico Norte (OTAN), quien tuvo tanta influencia durante la Guerra Fría cuando las Naciones Unidas, con frecuencia, estuvo eclipsada políticamente. Completan esa relación las Organizaciones No-Gubernamentales (ONGs) -sobre todo aquellas con una base internacional que hoy usan considerablemente a Naciones Unidas y a su vez.son ușadas por ésta-creándose así nuevos y variados tipos de relaciones.

La tercera percepción es que si el lenguaje de la Carta de Naciones Unidas es elocuente, elevado e inspirador en el enunciado de sus propósitos y principios, ello también resulta ser minucioso, prescriptivo y realista -en el sentido fuerte del término- en todo lo que respecta las reglas de operación del poder intemacional. Aunque mediatizadas, esas reglas se hacen sentir en el ámbito de la Organización. Los cambios en la agenda de Naciones Unidas son realizados por la inclusión o el olvido de ciertos temas y por el énfasis con que se los trata. Se produce así una especialización de tareas entre los distintos mecanismos multilaterales que nada tiene de neutra o de apolitica, porque es una división del trabajo basada en el poder.

Naciones Unidas fue concebida como la piedra de toque de la macroestructura internacional. En su origen, teniendo en cuenta los propósitos y principios que consagra, su Carta puede ser interpretada como un pacto "horizontal" entre Estados igualmente soberanos, aunque dispares en poder. La Carta debería así tener un rol verdaderamente constitutivo del orden internacional, basado en el principio de la responsabilidad colectiva por la paz. Desde 1945, empero, la realidad política ha demostrado ser muy difícil. Los bloques antagónicos del Este y Oeste obstaculizaron la observancia de los propósitos y principios de la Carta y la práctica de aquella responsabilidad colectiva.

Como concepción jurídica y política, el mecanismo de mantenimiento de la paz y de la seguridad permite hacer más ordenada, y de ende más previsible, la vida internacional, puesto que organiza la comunidad de 
Estados para armonizar sus acciones, resolver las controversias, disuadir las amenazas y penalizar cualquier agresión. Pero en San Francisco la entronización de la facultad de veto redujo esa concepción a ciertas realidades que tienen por emblema la preponderancia de los miembros permanentes del Consejo de Seguridad.

En ausencia de acuerdo entre los grandes, se paralizaba el Consejo. Más grave aún: la repetida utilización del veto garantiza, en la práctica, a los miembros permanentes inmunidad política y juridica respecto a la operación del mecanismo colectivo. Durante la Guerra Fría, en el impasse y consecuente intolerancia política del Consejo, proliferaron los acuerdos de seguridad regional. La Organización de Estados Americanos y el Tratado de Río de Janeiro fueron sus prototipos. Luego le siguieron la OTAN y otros pactos regionales. El espejo de esa imagen fue la constitución del Tratado de Varsovia.

Durante el periodo inicial de la Guerra Fría, la ascendencia occidental le permitía movilizar automáticamente la mayoría en todas las cuestiones de seguridad, asi como aquellas de carácter económico y social que pudiesen ser planteadas en términos ideológicos. Naciones Unidas se convirtió en un escenario para la guerra de propaganda, en perjuicio de sus funciones negociadoras y de foro para la implementación del mecanismo de seguridad colectiva. La Organización se descaracterizaba y, por largo tiempo, fue relegada al descrédito en lo que vendría a ser llamada la "crisis del multilateralismo".

Hoy día las premisas políticas, económicas y estratégicas que produjeron la Carta de Naciones Unidas y la Organización misma no tienen más vigencia. A excepción de enmiendas menores, la Carta es aún la de 1945 y refleja mayormente una situación intemacional ultrapasada. Las estructuras institucionales de Naciones Unidas están en parte atrofiadas y no responden a las necesidades actuales. Necesitan, por esa razón, ser repensadas y reorganizadas. La composición y los procedimientos arcaicos y de poca transparencia del Consejo de Seguridad son dos buenos ejemplos.

Pero la Carta, con su plasticidad interpretativa, puede convertirse en el vehículo de transición hacia la posible elaboración de un nuevo orden mundial equitativo. Las lecturas contemporáneas de la Carta propician concepciones y proposiciones variadas. Algunos llegan incluso a sobrevalorar el momento actual y a afirmar, sin capacidad crítica, que por fin el Consejo de Seguridad estaria funcionando de la manera con que habrian visualizado los redactores de la Carta. Otros ven a Naciones Unidas como un simple y limitado reflejo del esquema de fuerzas 
prevalecientes, como una organización fatalmente subordinada a la jerarquía entre los Estados. Aún hay quien la ve como una precursora de formas de supranacionalismo utópico.

Esas interpretaciones sirven de soporte ideológico y político para la articulación de visiones alternativas del futuro de las relaciones internacionales. Se intenta operacionalizar esos enfoques, ya sea con una lectura de la Carta como si fuera ella un documento inmutable en su letra y espíritu o con la proposición activa de su reforma para adaptarla a las realidades nuevas.

Como no hay un consenso firme sobre el contenido y las implicaciones políticas de esas nuevas realidades, crecen las polémicas y se imponen algunas aclaraciones:

-Primero: Naciones Unidas no puede ser, desde un punto de vista histórico y político, reducida a términos estáticos, ni sería posible confinarla a las dimensiones de un simple instrumento diplomático al servicio de un país o de un grupo de países. Naciones Unidas no es la comisión frente de las potencias dominantes, no se destina a avalar las acciones políticas y militares de ellas o, aún menos, no se resume a un escenario donde los países periféricos puedan expresar sus frustraciones.

-Segundo: las prerrogativas expresamente delimitadas del Consejo de Seguridad y de sus miembros permanentes les fueron específicamente atribuidas por el conjunto de los Estados miembros. No fueron, por lo tanto, autoatribuidas o el resultado de gestación espontánea, ni pueden, en rigor, ser expandidas autónomamente. -Tercero: Naciones Unidas no constituye, de ninguna manera, un gobiemo mundial. Carece de condiciones jurídicas y políticas para eso. Es una organización exclusivamente internacional, nunca supranacional, que depende de la voluntad política de sus Estados miembros y no se superpone a ellos.

Por otro lado, hay que destacar que cuando las grandes potencias recurren al Consejo to que es hoy día frecuente-lo hacen bajo la reserva expresa de que ese recurso no precluye la facultad de actuar unilateralmente o de hacerlo mediante coaliciones ad hoc que no están necesariamente bajo control de la Organización. Para esas grandes potencias, hoy el Consejo funciona normalmente: la facultad de veto se mantiene aunque sea raramente ejercida, pero ella no es la única opción para la solución de las crisis.

La multiplicidad de tareas recientemente atribuidas al Consejo de Seguridad ha facilitado la expansión de sus actividades. El Consejo es 
un organismo ejecutivo, eñ la medida en que tiene, en situaciones especificas, la facultad de determinar acciones concretas y obligatorias para el mantenimiento de la paz y de la seguriơad intemacionales, y de vigilar la implementación de esas medidas. El carácter del Consejo puede también ser: diplomático, en la medida en que permite la producción de soluciones negociadas entre las Partes; conminatorio, cuando las soluciones se basan en el Capitulo VII de la Carta; y casi-judicial, cuando asume el rol de árbitro de situaciones sobre las cuales puede decidir, sin posibilidad de revisión del mérito jurídico o político.

En. verdad, en los últimos años el Consejo de Seguridad bajo el liderazgo de sus miembros permanentes $y$, en particular, de los tres occidentales- ha empezado a ampliar sus atribuciones jurisdiccionales. Se sostiene para ello, que esa ampliación no sería contraria a la "letra" o al "espiritu" del texto constitucional que constituye la Carta, y se intenta así usar a Naciones Unidas como una instancia legitimadora para la mantención del status quo. Bajo la influencia de esas ideas, el Consejo ha querido atribuirse funciones antes normativas del orden internacional, debido a la creación de precedentes como fuentes de case law constitutivos del orden mundial que son sustraídos de la comunidad internacional más amplia. Estas acciones innovadoras del Consejo presuponen "interpretaciones creativas" de la Carta que funcionan como "reformas blancas" y reinterpretan la Carta sin pasar por el proceso traumático de la adopción de enmiendas.

En ese sentido, se da un énfasis renovado a las cuestiones de "diplomacia preventiva", "peace-making" "e "imposición de la paz". A pesar de las dificultades financieras y operacionales de Naciones Unidas, se llegó a vislumbrar, hace menos de dos años, la idea de dotar a Naciones Unidas directamente con una especie de "ejército permanente" para despliegue inmediato en situaciones de crisis internacional. La expectativa ingenua de que Naciones Unidas pudiese desempeñar un rol activo como guardián militarizado de la paz, practicando operaciones de "nueva generación" mediante la irónica "imposición de la paz por la fuerza", fue rápidamente disipada cuando se percibieron los riesgos militares inherentes a las crisis que ocurrieron en la post-Guerra Fría. De la euforia por aparentes éxitos preliminares en Somalía y en la antigua Yugoslavia, pronto se pasó a la constatación sobria del impasse en que se encontraba Naciones Unidas en aquellas regiones, muchas veces ubicada en medio de atascaderos políticos y militares y hostilizada por todas las partes en conflicto. 
En realidad Naciones Unidas vive una etapa crucial, no solamente en términos de la hipertrofia del Consejo de Seguridad hasta el año 1993, sino también ahora por una aguda percepción de la incapacidad de la Organización para resolver conflictos verdaderamente espinosos. Todo eso transcurre contra el telón de fondo de la crisis financiera de la Organización que se originó en el incumplimiento de sus mayores contribuyentes. Hay, además, otros problemas centrales como la necesidad de lograr un mayor equilibrio entre los organismos principales de la Organización, de crear instancias para acompañar el rol creciente activismo del Secretariado, y de establecer un mecanismo de equilibrio entre el peso político de los países industrializados y la menor capacidad parlamentaria de los numerosos países en desarrollo y países no-alineados.

La baja representatividad y efectividad del Consejo como organismo legítimamente reconocido por el universo de los miembros de Naciones Unidas se debe en parte, por ejemplo, a la dificultad del Movimiento No-Alineado (MNOA) para establecer su identidad y su propósito de acción dentro de la Organización en la post-Guerra Fría. Aunque disponga de una representación numérica que sería suficiente para, en el mínimo, bloquear la adopción de resoluciones cruciales en Consejo de Seguridad, el llamado caucus del MNOA tiene dificultades en contraponerse a la actuación asertiva de los miembros permanentes del Consejo cuando estos últimos intentan afirmar sus intereses.

En último término, ese tipo de situación refleja, sobre el plano de la dirección de los trabajos del Consejo, la contradicción entre los anhelos de una mayor democratización y las necesidades de una mayor efectividad en la composición de ese organismo. Si es lícito defender, de un lado, que todos los países tienen derechos iguales de acceso a una silla del Consejo, es igualmente impositivo reconocer que, por ejemplo, la ausencia de paises como Brasil o India en deliberaciones de cuestiones cruciales para la paz y la seguridad compromete el equilibrio político que debería servir de base a la evaluación y a la actuación de Naciones Unidas.

La pérdida de peso relativo de la Asamblea General es otro factor de preocupación. La Asamblea está, en verdad, presionada por el universalismo de su composición y la extensión de su agenda de más de 120 temas. Eso no significa que se deba aceptar pasivamente el argumento que la eficacia de un organismo de composición restrictiva sería preferible, como factor de legitimación, a la representatividad de un organismo universal. 


\section{ESTUDIOS INTERNACIONALES}

El Consejo de Seguridad es justamente un organismo de composición extremadamente restringida $y$, por ende, se beneficia de una agilidad decidora, pero se resiente, por otro lado, de la falta de una legitimidad representativa. La Asamblea, a su vez, por su composición universal, es el organismo más democrático y representativo de la comunidad internacional contemporánea, pero se ha mostrado inoperante, en los últimos años, en el tratamiento de las cuestiones cruciales bajo su competencia.

Así, por un lado, la reestructuración del Consejo de Seguridad -sobre la cual la posición brasileña es ampliamente conocida-resulta un imperativo político. Del otro, la revitalización de la Asamblea es igualmente imprescindible. Brasil siempre ha estado en la delantera de la promoción de los idearios de desarrollo, desarme y democratización de las relaciones internacionales. El verdadero equilibrio, cualitativo y operacional, entre esos dos organismos de apoyo para las relaciones internacionales garantizaria a todos los Estados una participación equitativa en la conformación del nuevo orden internacional. Esas banderas son fundamentales para el destino de Naciones Unidas y, en último término, para la inserción internacional de Brasil. 